\section{CLINICAL COURSE, PROGNOSIS AND HEALTH CARE INSURANCE OF A COHORT OF LOW BIRTH WEIGHT INFANTS DISCHARGED IN KANGAROO POSITION}

\author{
N. Charpak ${ }^{1,2}$, A. Lombo 3
}

${ }^{1}$ Kangaroo Foundation, ${ }^{2}$ Integral Kangaroo Mother Care Program, San Ignacio Teaching Hospital, Javeriana University, Bogota, ${ }^{3}$ Integral Kangaroo Mother Care Program, Clinica del Prado, Medellin, Colombia

Objective: Clinical course and prognosis at one year of a LBWI cohort cared in a ambulatory kangaroo mother care program (KMCP) according to health care insurances: Private prepaid plan (high incomeHI) Contributive plan (middle and low-MLI) and subsidized plan (very low-VLI).

Methods: Prospective LBWI cohort discharged in a KMCP with follow-up until 12 months to determine survival, growth, development and morbidity. Same guidelines were implemented in all types of health care insurance.

Results: On 6889 infants admitted: 4\% were from $\mathrm{HI}, 90 \%$ from $\mathrm{MLI}$ and $6 \%$ from VLI plans. Overall mortality was $1.6 \%$ for the three groups. While $100 \%$ of deaths for $\mathrm{HI}$ infants occur during hospitalization, $24 \%$ and $45.5 \%$ in $\mathrm{MLI}$ and VLI groups were at home. Frequency of infectious diseases was markedly increased in VLI $(25.8 \%$ in $\mathrm{HI}$ vs. $50 \%$ in VLI). Nearly half of patients in MLI and VLI received exclusive breastfeeding up to term versus $4.6 \%$ in $\mathrm{HI}$. Weight, length and head circumference at birth and at one year of corrected age didn't show great variation between groups. Cerebral palsy at one year was increased in VLI group: $3.5 \%$ vs. $1.8 \%$ in $\mathrm{HI}$ and $1.3 \%$ in MLI. Mean developmental coefficient at 12 months didn't show variation between groups.

Conclusion: Results of KMC implementation are similar independently of the type of health care insurance. Mortality and anthropometric indices during follow up were the same, but morbidity was increased in VLI group. KMC rules rigorously applied provide same benefits to all LBWI in Colombia regardless of socio economic level.

\section{EVIDENCE-BASED GUIDELINES FOR THE USE OF THE KANGAROO MOTHER CARE (KMC) METHOD IN STABLE LBW INFANTS IN COLOMBIA}

\author{
J.G. Ruiz-Pelaez ${ }^{1,2}$, N. Charpak ${ }^{2}$
}

${ }^{1}$ Clinical Epidemiology and Biostatistics, Javeriana University, ${ }^{2}$ Kangaroo Foundation, Bogota, Colombia

Background: KMC method is a set of interventions for providing appropriate health care to preterm and/or LBW infants, based on the kangaroo position. Since it was first described (Rey 1978) considerable variability has developed. There is a need to standardize the intervention, based on scientific evidence that supports its benefits and limitations.

Objective: Develop recommendations about characterization and proper use of the KMC method, and to support each assertion with a systematic review of evidence.

Design: A Multidisciplinary group (authorities, users, parents of patients, methodological and content experts) worked between 2005 and 2007. After defining terms and characterizing components of the intervention, a systematic review of literature was conducted for identifying and appraising the evidence regarding the effects, risks, benefits and limitations of each component. Evidence based assertions were discussed until consensus was achieved and were evaluated by external peers.

Results: Standardized definitions of KMC and its component, variants, target population, indications and precautions were produced. An evidence-based foundation for each component was developed, identifying strength of evidence, knowledge gaps, areas of controversy and needs for further research. Discussion of basic aspects and results of relevant research were added to each recommendation together with descriptions of the appropriate implementation of KMC.

Conclusions: Although sound guidelines should be tailored to local needs and conditions, and this particular exercise is focused in the Colombian situation, it is reasonable to expect that many of the recommendations and certainly most of the evidence appraised will be a useful input for guidelines development elsewhere. 